\section{Aktualisiertes Fachwissen in prägnanter Form}

$D_{\mathrm{re}}^{\mathrm{ic}}$ Checklisten sind seit Jahrzehnten routinemäßiger Kitteltaschen-Begleiter von Medizinstudenten und Assistenten in der Fachausbildung, weil sie praxisnahes Fachwissen kompakt in "griffiger" Form bereithalten. Auch die Checkliste HNOHeilkunde ist in diesem positiven Sinne „in die Jahre gekommen“ und erscheint als komplett überarbeitete und aktualisierte Fassung nun schon in der 5. Auflage.

Die Herausgeber haben auf knapp 600 Seiten im DIN-A-5-Format eine umfassende und aktuelle Darstellung der gesamten HNO-Heilkunde untergebracht - unter Berücksichtigung der Anforderungen der Europäischen Facharztnorm (UEMS) und mit zum Teil ausgezeichnetem Bildmateri- al. Nach einem einleitenden „Grundlagenteil" mit kurzgefassten Angaben zu Anatomie, Physiologie und Diagnostik folgt ein Kapitel zu den wichtigsten Leitsymptomen des Fachgebietes, das in Algorithmenform abgefasst ist. Anschließend werden die einzelnen „Organe“ der HNO-Heilkunde systematisch und auf dem neuesten Stand „abgearbeitet“. Der letzte Teil widmet sich den operativen Verfahren des Fachgebietes, und auch hier werden die wesentlichen Eingriffe berücksichtigt.

Sehr gut haben mir persönlichdie Beiträge zu CI und implantierbaren Hörgeräten gefallen, während die Texte zu transantraler Kieferhöhlenoperation und BellocqTamponade für mein Dafürhalten gekürzt

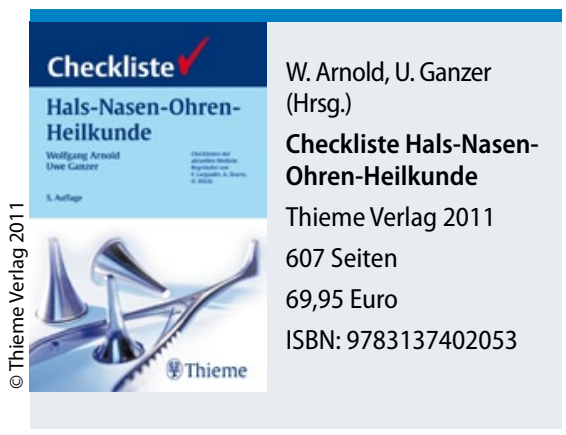

werden könnten, da diese Techniken heute eine doch eher nachgeordnete Bedeutung haben.

Insgesamt erhält der Leser mit der Checkliste HNO-Heilkunde ein über zwei Jahrzehnte gewachsenes und ausgereiftes Taschenbuch unseres Fachgebietes.

Prof. Dr. med. Gerhard Grevers

\title{
Kompendium der Tracheotomie
}

D iese Operation gereicht dem Arzt zur Ehre und macht ihn gottähnlich", schrieb der berühmte Wundarzt Fabricius über die Tracheotomie. So vermessen wollen wir in der modernen Medizin nicht sein. Die selbstständige Durchführung einer Tracheotomie war allerdings Anfang der 70er-Jahre Voraussetzung damit man als HNO-Weiterbildungsassistent im klinischen Nachtdienst eingesetzt werden durfte.

Die Tracheotomie pendelt heute zwischen HNO und Intensivmedizin, trotzdem sollte der HNO-Arzt diesen Eingriff nicht ausschließlich anderen Fachrichtungen überlassen. Unser Fachwissen spielt eine erhebliche Rolle bei der Vermeidung und Therapie von Komplikationen. Das von Klemm und Novak vorgelegte Kompendium erfüllt alle Voraussetzungen für einen vollständigen Überblick bei allen Facetten der Tracheotomie. Eine wichtige Rolle spielt die Trachealchirurgie mit dem diffizilen Airway-Management, Stents etc. Auch die Rehabilitation nach schwerer Grunderkrankung wird ausführlich beschrieben.

Mit seiner interdisziplinären Sichtweise auf Analyse, Vermeidung und Therapie schwerer Früh- und Spätkomplikationen, einschließlich logopädischer Behandlung und einer speziellen Haut-und Schleimhautpflege des Tracheostomas, bietet das vorgelegte Kompendium eine Fülle von

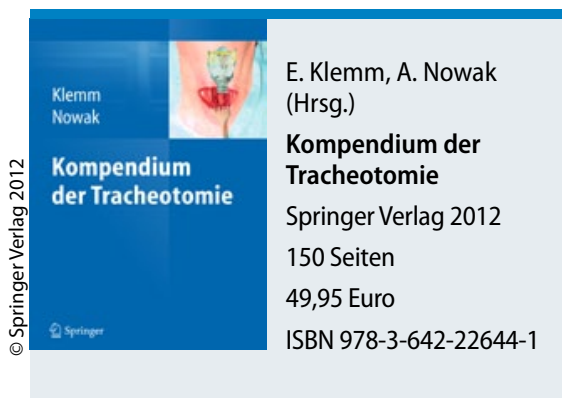

Fakten und Anregungen für unser Fach. Zu erwähnen ist das kompetente Autorenteam der beiden Herausgeber. Der Buchinhalt beruht auf den Ergebnissen des jährlich in Dresden stattfindenden TracheotomieTages. Die Gestaltung des Buches mit hervorragenden Abbildungen ist vorbildlich.

Dr. med. Dieter Leithäuser

\section{Interessenkonflikte in der Medizin}

— inem Arzt muss man vertrauen kön-

- nen. Jeder Patient möchte sicher sein, dass das erste Interesse des Arztes das Patientenwohl ist - und nichts anderes. Welche Rolle spielen jedoch Interessenkonflikte für die Behandlungsentscheidung des Mediziners? Was sind Interessenkonflikte? Wie stellt man sie fest? Über welche psychologischen Mechanismen beeinflussen sie Urteilsvermögen und Entscheidungen und wie sind Interessenkonflikte von Korruption abzugrenzen?
Das neue Buch „Interessenkonflikte in der Medizin" beantwortet diese und andere Fragen. Es stellt den aktuellen Stand der internationalen Diskussion sowie die innerdeutsche Situation dar, zeigt Hintergründe und Handlungsoptionen auf und bietet Empfehlungen zum Umgang mit Interessenkonflikten. Zudem geht es auf spezifische Interessenkonflikte in den unterschiedlichen Bereichen des Gesundheitswesens ein: in der Patientenversorgung, der Forschung, der ärztlichen Aus,

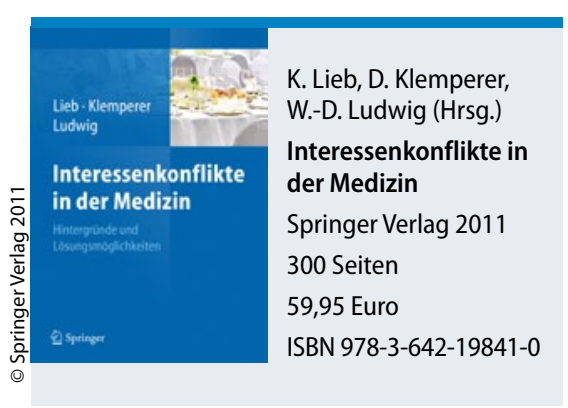

Weiter- und Fortbildung sowie in medizinischen Fachzeitschriften und im $\mathrm{Me}$ dizinjournalismus. 